\title{
Experimental Study of the Crystal Habit of High Explosive Octahydro-1,3,5,7-tetranitro-1,3,5,7- tetrazocine (HMX) in Acetone and Dimethyl sulfoxide
}

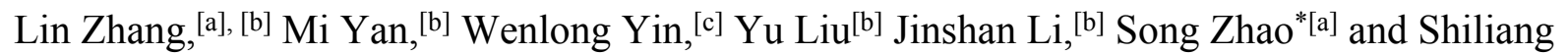
Huang ${ }^{*}[b]$

[a] School of Chemical Engineering and Technology, Tianjin University, Tianjin, 300072, PR China

${ }^{[b]}$ Institute of Chemical Materials, China Academy of Engineering Physics, Mianyang, 621900, PR China

[c] Sichuan New Material Center, Chengdu, 030051, PR China 
Supplementary information

\section{List of Contents}

Part 1. The measurement of the thickness for the HMX crystals grown in acetone and DMSO.

Part 2. Area measurement of the facets of the HMX crystals grown in acetone and DMSO. 
Part 1. The measurement of the thickness of the HMX crystals grown in acetone and DMSO.

1.1 The HMX crystals grown in acetone.
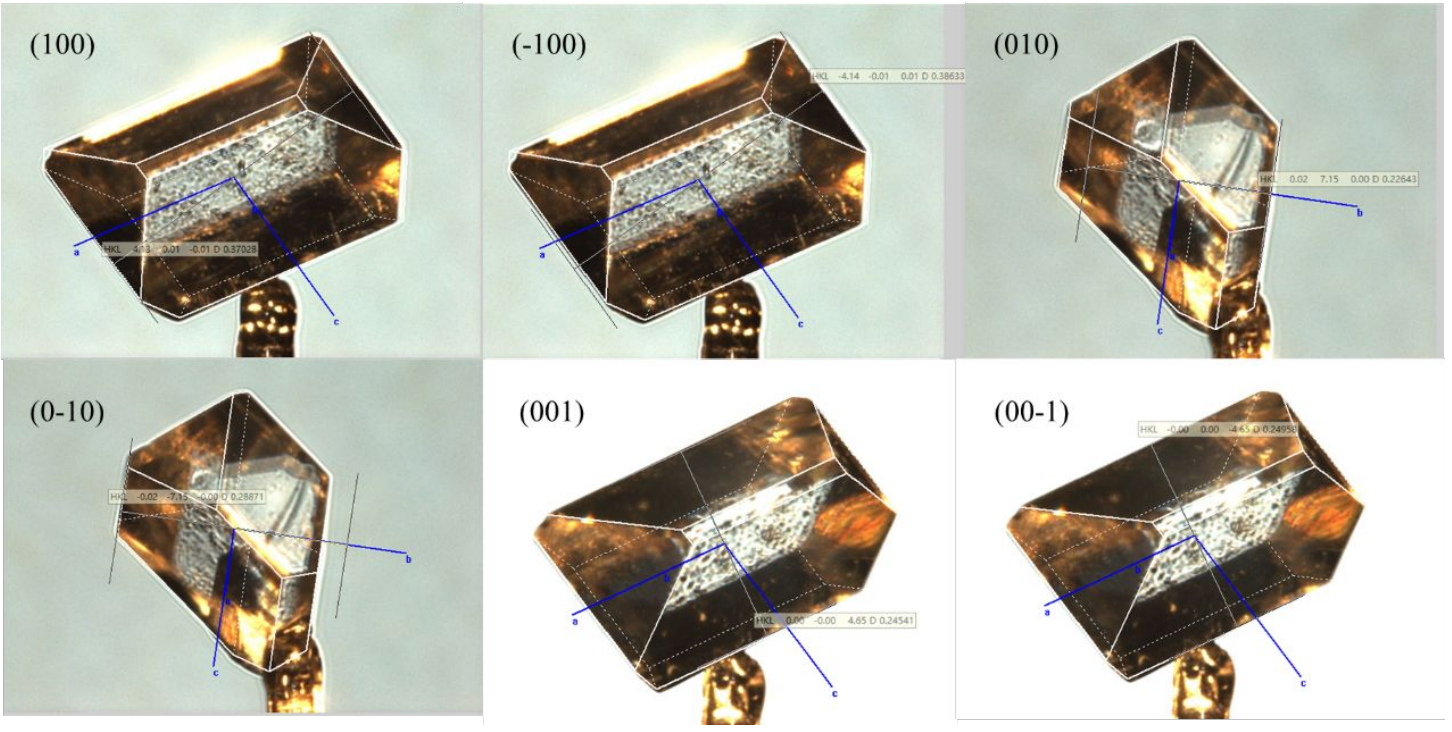

Figure S1. The crystal thickness measurement of Sample 1 along the $a, b$ and $c$-axis.

Table S1. The thickness of sample 1 along the $a, b$ and $c$-axis.

\begin{tabular}{|c|c|c|c|c|c|c|}
\hline Sample & \multicolumn{5}{|c|}{ Distance from the facet to the center of the crystal, $\mathbf{D}$, in the unit of $\mathrm{mm}$} \\
\hline & $(100)$ & $(-100)$ & $(010)$ & $(0-10)$ & $(001)$ & $(00-1)$ \\
\hline $\mathbf{1}$ & 0.370 & 0.386 & 0.226 & 0.289 & 0.245 & 0.250 \\
\hline
\end{tabular}
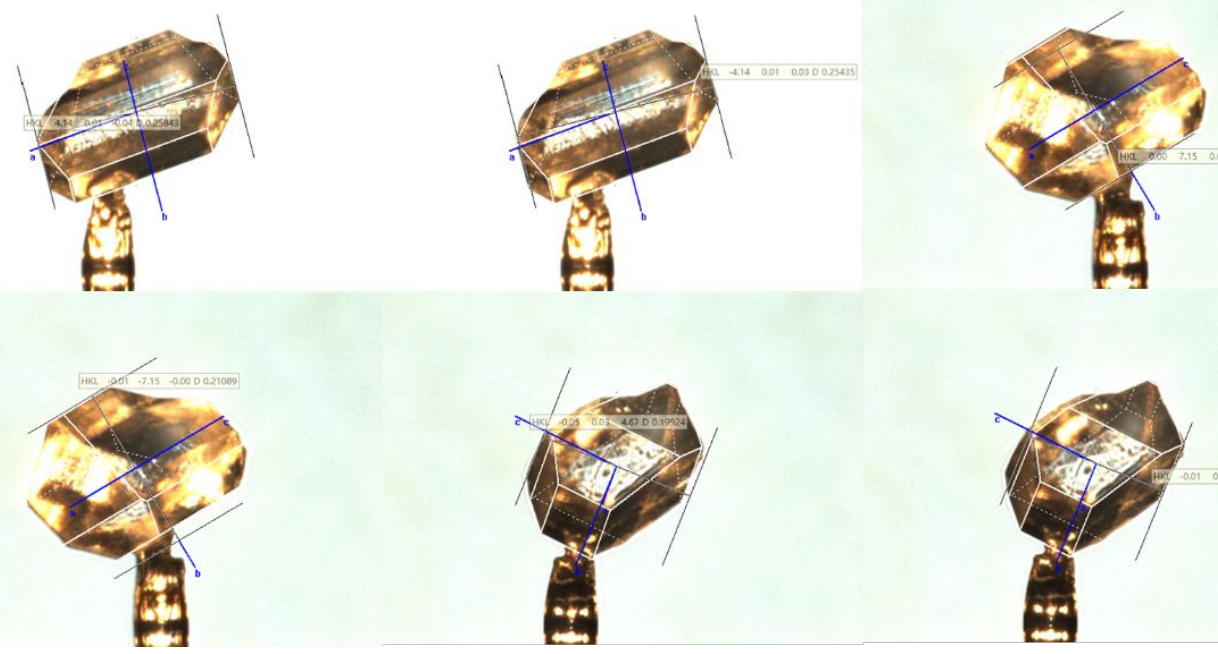

Figure S2. The crystal thickness measurement of Sample $\mathbf{2}$ along the $a, b$ and $c$-axis.

Table S2. The thickness of sample 2 along the $a, b$ and $c$-axis.

\begin{tabular}{|c|c|c|c|c|c|c|}
\hline Sample & \multicolumn{5}{|c|}{ Distance from the facet to the center of the crystal, D, in the unit of $\mathrm{mm}$} \\
\hline & $(100)$ & $(-100)$ & $(010)$ & $(0-10)$ & $(001)$ & $(00-1)$ \\
\hline $\mathbf{2}$ & 0.258 & 0.254 & 0.174 & 0.211 & 0.199 & 0.183 \\
\hline
\end{tabular}




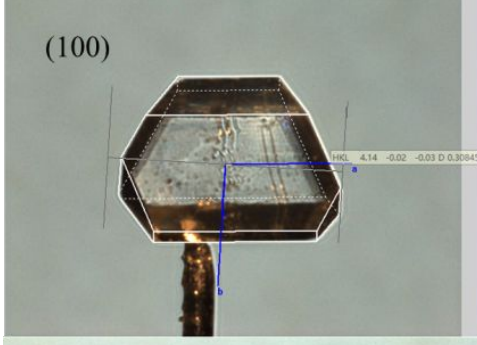

$(-100)$

$(0-10)$

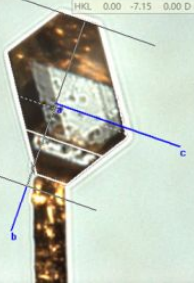

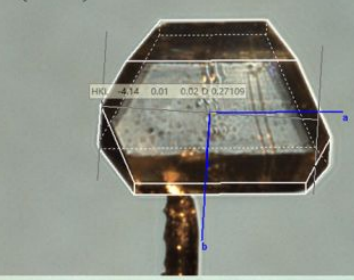

$(001)$

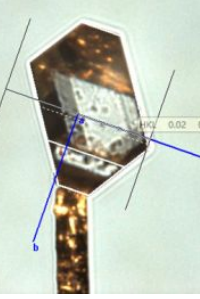

(010)

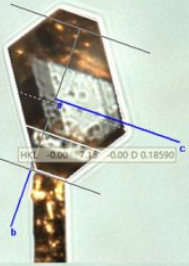

$(00-1)$

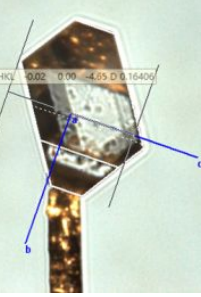

Figure S3. The crystal thickness measurement of Sample 3 along the $a, b$ and $c$-axis.

Table S3. The thickness of sample 3 along the $a, b$ and $c$-axis.

\begin{tabular}{|c|c|c|c|c|c|c|}
\hline Sample & \multicolumn{5}{|c|}{ Distance from the facet to the center of the crystal, D, in the unit of $\mathrm{mm}$} \\
\hline & $(100)$ & $(-100)$ & $(010)$ & $(0-10)$ & $(001)$ & $(00-1)$ \\
\hline 3 & 0.308 & 0.271 & 0.186 & 0.220 & 0.192 & 0.164 \\
\hline
\end{tabular}

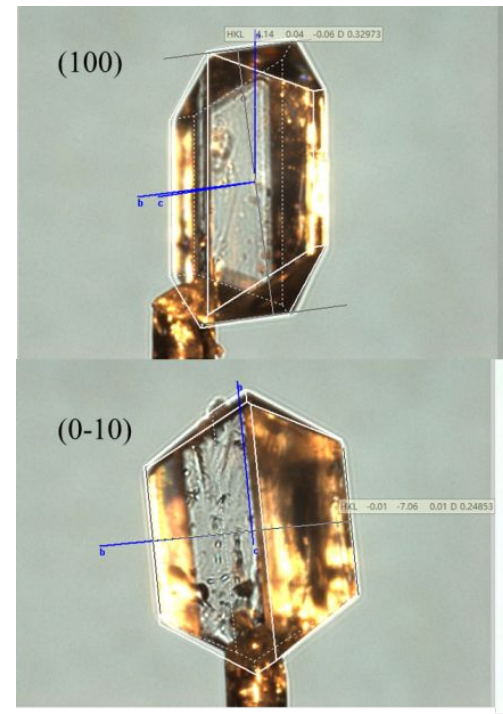

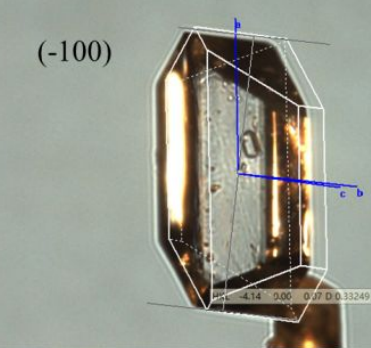

(001)

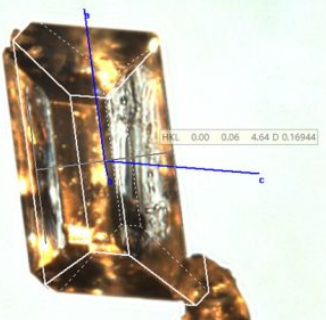

(010)

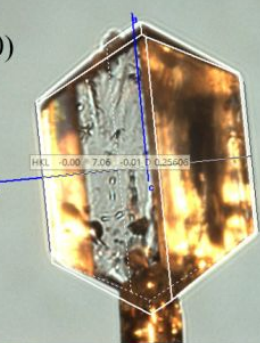

$(00-1)$

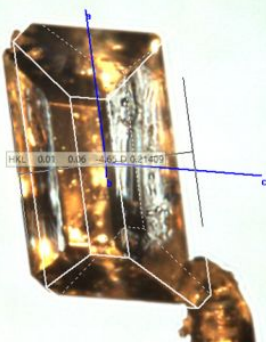

Figure S4. The crystal thickness measurement of Sample 4 along the $a, b$ and $c$-axis.

Table S4. The thickness of sample 4 along the $a, b$ and $c$-axis.

\begin{tabular}{|c|c|c|c|c|c|c|}
\hline Sample & \multicolumn{5}{|c|}{ Distance from the facet to the center of the crystal, D, in the unit of $\mathrm{mm}$} \\
\hline & $(100)$ & $(-100)$ & $(010)$ & $(0-10)$ & $(001)$ & $(00-1)$ \\
\hline $\mathbf{4}$ & 0.330 & 0.332 & 0.256 & 0.249 & 0.169 & 0.214 \\
\hline
\end{tabular}




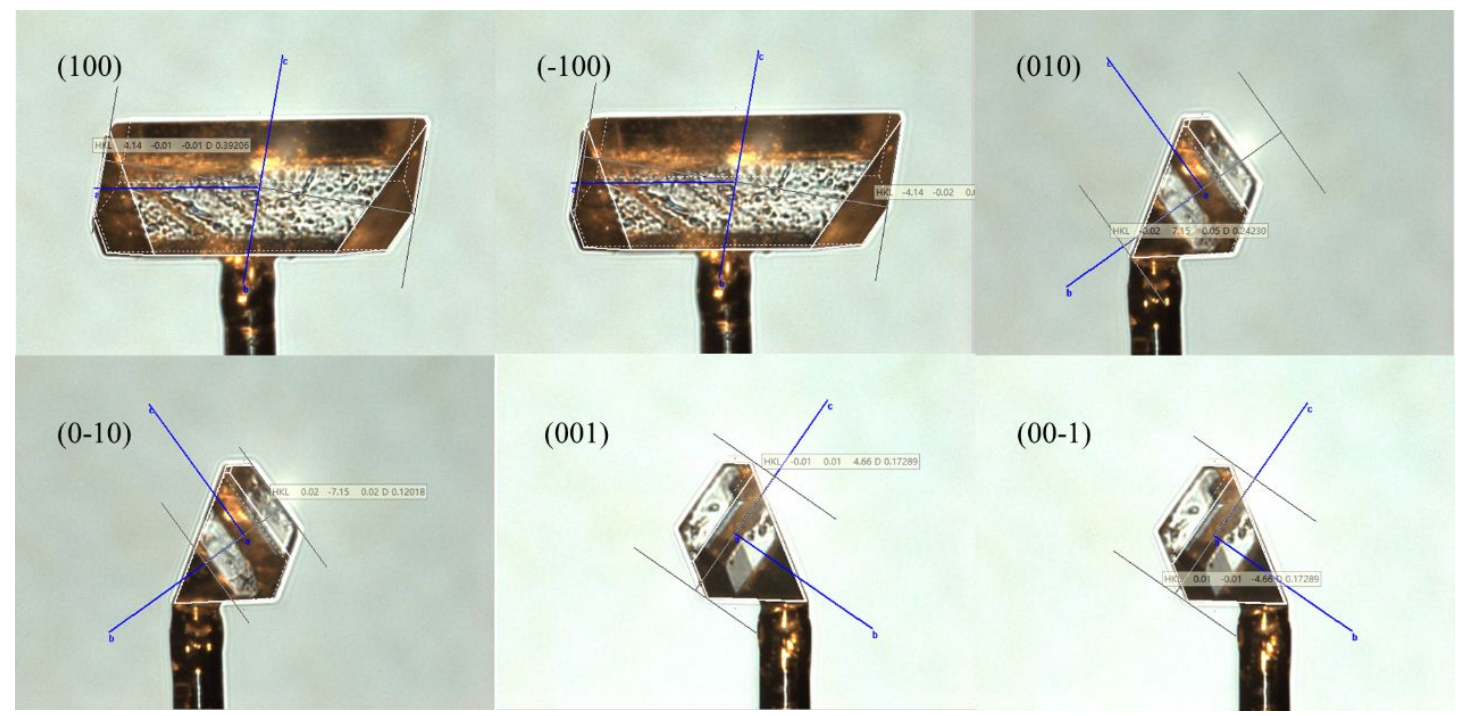

Figure S5. The crystal thickness measurement of Sample 5 along the $a, b$ and $c$-axis.

Table S5. The thickness of sample 5 along the $a, b$ and $c$-axis.

\begin{tabular}{|c|c|c|c|c|c|c|}
\hline Sample & \multicolumn{5}{|c|}{ Distance from the facet to the center of the crystal, D, in the unit of $\mathrm{mm}$} \\
\hline & $(100)$ & $(-100)$ & $(010)$ & $(0-10)$ & $(001)$ & $(00-1)$ \\
\hline $\mathbf{5}$ & 0.392 & 0.386 & 0.242 & 0.120 & 0.173 & 0.173 \\
\hline
\end{tabular}

$(100)$

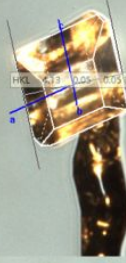

$(0-10)$

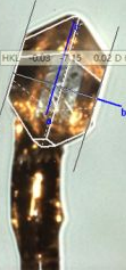

$(-100)$

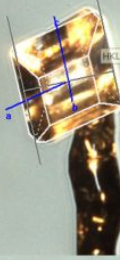

$(001)$

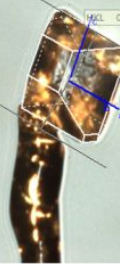

$(010)$

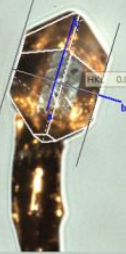

$(00-1)$

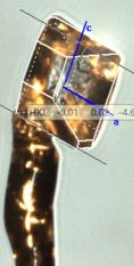

Figure S6. The crystal thickness measurement of Sample 6 along the $a, b$ and $c$-axis.

Table S6. The thickness of sample 6 along the $a, b$ and $c$-axis.

\begin{tabular}{|c|c|c|c|c|c|c|}
\hline Sample & \multicolumn{5}{|c|}{ Distance from the facet to the center of the crystal, D, in the unit of $\mathrm{mm}$} \\
\hline & $(100)$ & $(-100)$ & $(010)$ & $(0-10)$ & $(001)$ & $(00-1)$ \\
\hline $\mathbf{6}$ & 0.124 & 0.113 & 0.111 & 0.107 & 0.136 & 0.112 \\
\hline
\end{tabular}


$(100)$

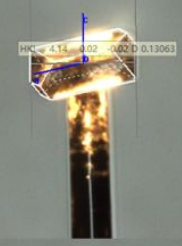

$(0-10)$

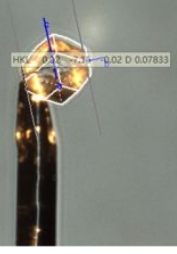

$(-100)$

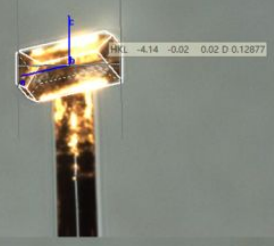

$(001)$

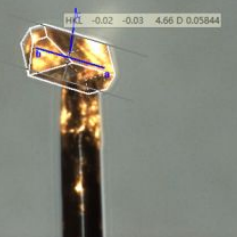

(010)

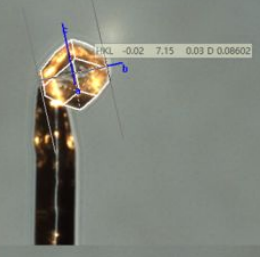

$(00-1)$

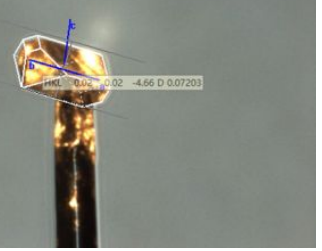

Figure S7. The crystal thickness measurement of Sample 7 along the $a, b$ and $c$-axis.

Table S7. The thickness of sample 7 along the $a, b$ and $c$-axis.

\begin{tabular}{|c|c|c|c|c|c|c|}
\hline Sample & \multicolumn{5}{|c|}{ Distance from the facet to the center of the crystal, D, in the unit of $\mathrm{mm}$} \\
\hline $\mathbf{7}$ & 0.131 & 0.129 & 0.086 & 0.078 & 0.058 & 0.072 \\
\hline
\end{tabular}

(100)

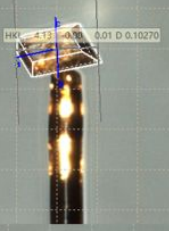

$(0-10)$

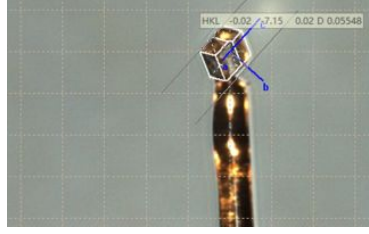

$(-100)$

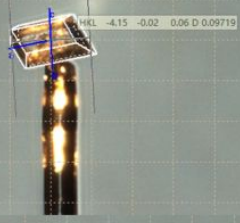

$(001)$

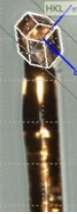

$(010)$

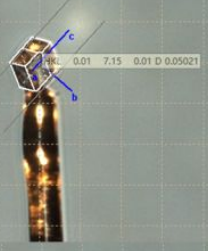

(00-1)

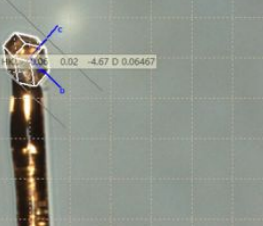

Figure S8. The crystal thickness measurement of Sample 8 along the $a, b$ and $c$-axis.

Table S8. The thickness of sample $\mathbf{8}$ along the $a, b$ and $c$-axis.

\begin{tabular}{|c|c|c|c|c|c|c|}
\hline Sample & \multicolumn{5}{|c|}{ Distance from the facet to the center of the crystal, D, in the unit of $\mathrm{mm}$} \\
\hline & $(100)$ & $(-100)$ & $(010)$ & $(0-10)$ & $(001)$ & $(00-1)$ \\
\hline $\mathbf{8}$ & 0.103 & 0.097 & 0.050 & 0.055 & 0.049 & 0.065 \\
\hline
\end{tabular}


Table S9. The summary of the thickness of the HMX crystals grown in AC along the $a$ (D1), $b$ (D2) and $c$-axis (D3) and their ratios.

\begin{tabular}{|c|c|c|c|c|c|}
\hline Sample & \multicolumn{3}{|c|}{ Thickness (mm)* } & \multicolumn{2}{c|}{ Ratio } \\
\hline & D1 & D2 & D3 & D1 : D3 & D2 : D3 \\
\hline $\mathbf{1}$ & 0.757 & 0.515 & 0.495 & 1.53 & 1.04 \\
\hline $\mathbf{2}$ & 0.513 & 0.385 & 0.383 & 1.34 & 1.01 \\
\hline $\mathbf{3}$ & 0.580 & 0.405 & 0.356 & 1.63 & 1.14 \\
\hline $\mathbf{4}$ & 0.662 & 0.505 & 0.384 & 1.73 & 1.32 \\
\hline $\mathbf{5}$ & 0.778 & 0.363 & 0.346 & 2.25 & 1.05 \\
\hline $\mathbf{6}$ & 0.237 & 0.218 & 0.248 & 0.95 & 0.88 \\
\hline $\mathbf{7}$ & 0.259 & 0.164 & 0.131 & 1.99 & 1.26 \\
\hline $\mathbf{8}$ & 0.200 & 0.106 & 0.114 & 1.76 & 0.93 \\
\hline \multicolumn{3}{|c|}{ Average } & 1.65 & 1.08 \\
\hline
\end{tabular}

* D1 $=\mathbf{D}(100)+\mathbf{D}(-100), \mathbf{D} 2=\mathbf{D}(010)+\mathbf{D}(0-10)$ and $\mathbf{D 3}=\mathbf{D}(001)+\mathbf{D}(00-1)$

Table S10. The thickness of the HMX crystals grown in acetone along the facet directions, in the unit of $\mathrm{mm}$.

\begin{tabular}{|l|c|c|c|c|c|c|}
\hline & $(\mathbf{0 1 1})$ & $\mathbf{( 0 1 - 1 )}$ & $\mathbf{( 1 1 0 )}$ & $\mathbf{( 1 - 1 0 )}$ & $\mathbf{( 0 2 0})$ & $(\mathbf{1 0 - 1})$ \\
\hline $\mathbf{1}$ & 0.507 & 0.343 & 0.707 & 0.613 & 0.484 & - \\
\hline $\mathbf{2}$ & 0.377 & 0.218 & 0.493 & 0.420 & 0.382 & - \\
\hline $\mathbf{3}$ & 0.356 & 0.243 & 0.540 & 0.465 & 0.404 & - \\
\hline $\mathbf{4}$ & 0.279 & 0.385 & 0.557 & 0.604 & 0.499 & - \\
\hline $\mathbf{5}$ & 0.236 & 0.338 & 0.690 & 0.671 & 0.371 & 0.861 \\
\hline $\mathbf{6}$ & 0.211 & 0.215 & 0.211 & 0.209 & 0.215 & 0.347 \\
\hline $\mathbf{7}$ & 0.119 & 0.106 & 0.230 & 0.225 & 0.173 & 0.251 \\
\hline $\mathbf{8}$ & 0.082 & 0.108 & 0.150 & 0.192 & 0.107 & 0.141 \\
\hline
\end{tabular}

\subsection{The HMX crystal grown in DMSO.}

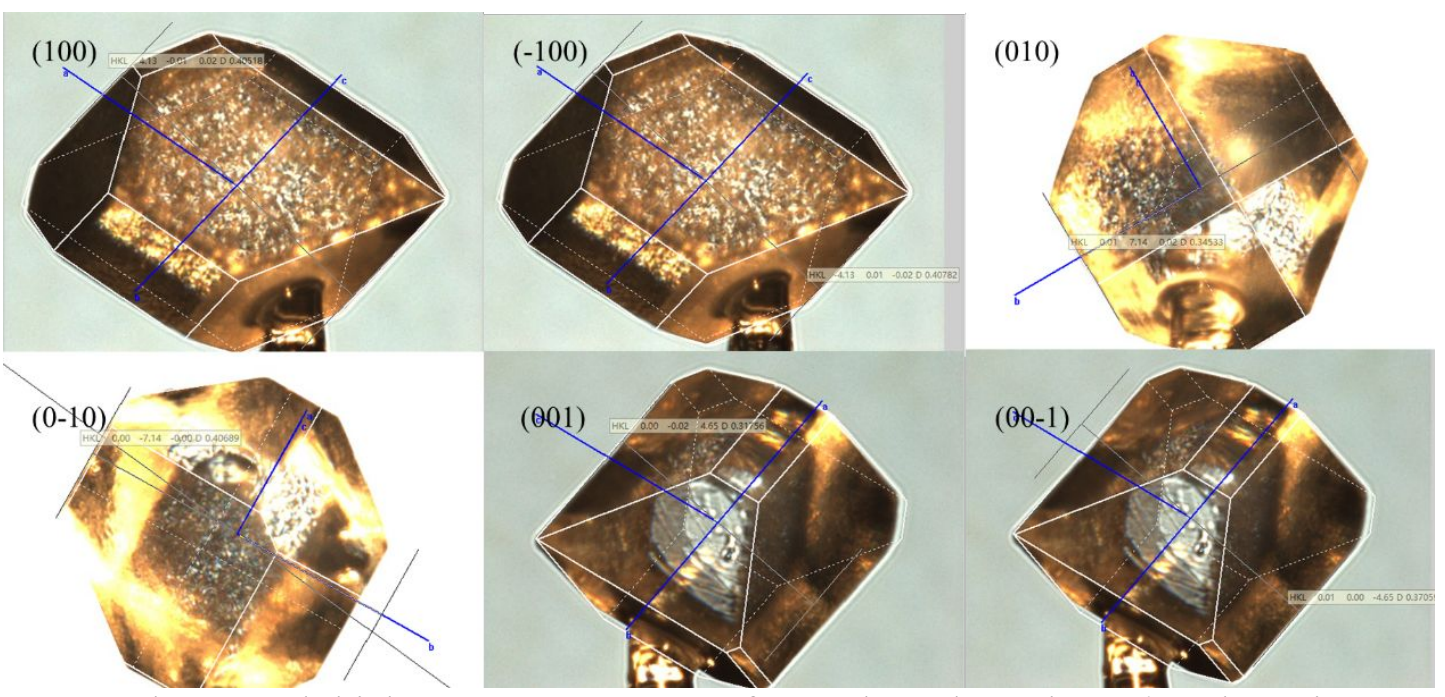

Figure S9. The crystal thickness measurement of Sample 1 along the $a, b$ and $c$-axis. 
Table S11. The thickness of sample 1 along the $a, b$ and $c$-axis.

\begin{tabular}{|c|c|c|c|c|c|c|}
\hline Sample & \multicolumn{5}{|c|}{ Distance from the facet to the center of the crystal, D, in the unit of $\mathrm{mm}$} \\
\hline & $(100)$ & $(-100)$ & $(010)$ & $(0-10)$ & $(001)$ & $(00-1)$ \\
\hline $\mathbf{1}$ & 0.405 & 0.408 & 0.345 & 0.407 & 0.318 & 0.371 \\
\hline
\end{tabular}
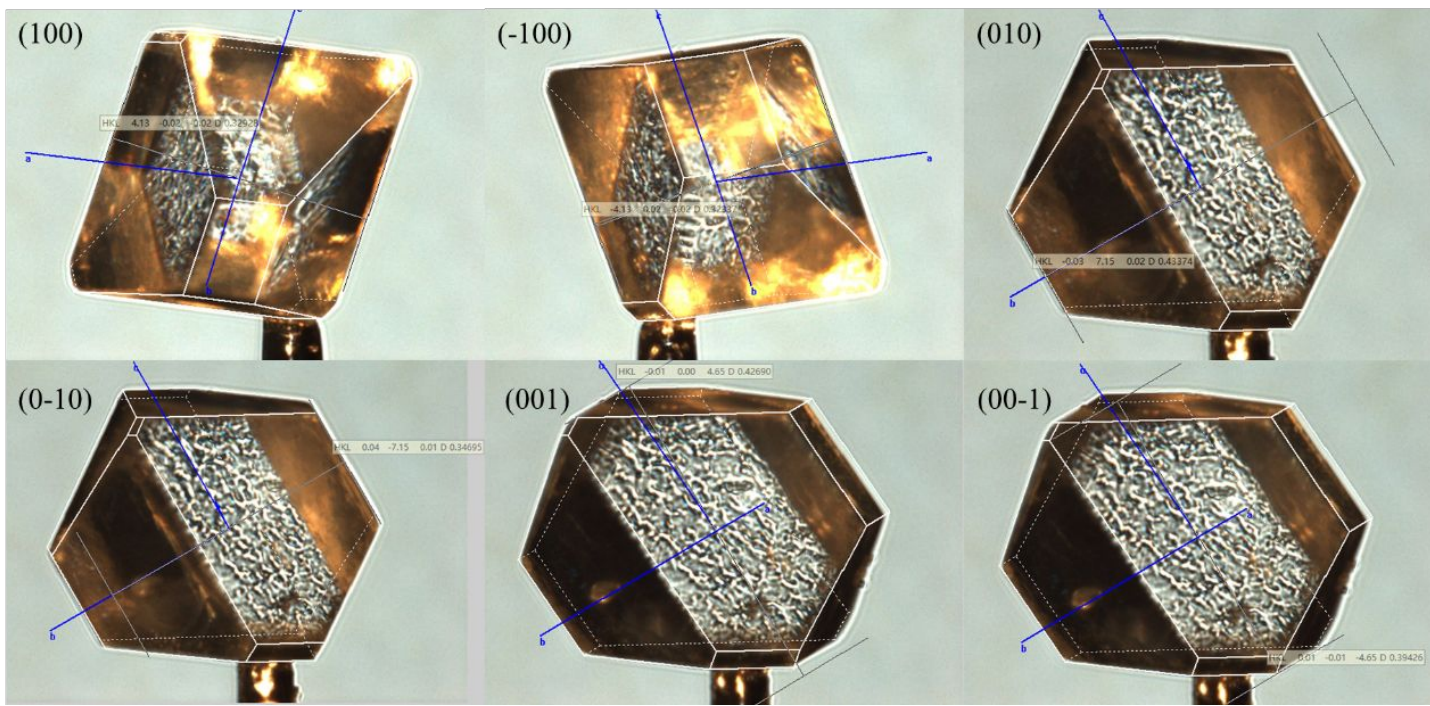

Figure S10. The crystal thickness measurement of Sample 2 along the $a, b$ and $c$-axis.

Table S12. The thickness of sample 2 along the $a, b$ and $c$-axis.

\begin{tabular}{|c|c|c|c|c|c|c|}
\hline Sample & \multicolumn{5}{|c|}{ Distance from the facet to the center of the crystal, D, in the unit of $\mathrm{mm}$} \\
\hline & $(100)$ & $(-100)$ & $(010)$ & $(0-10)$ & $(001)$ & $(00-1)$ \\
\hline $\mathbf{2}$ & 0.329 & 0.323 & 0.434 & 0.347 & 0.427 & 0.394 \\
\hline
\end{tabular}

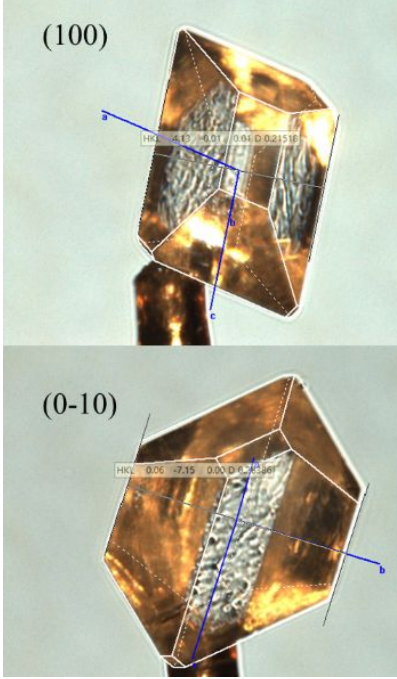

$(-100)$

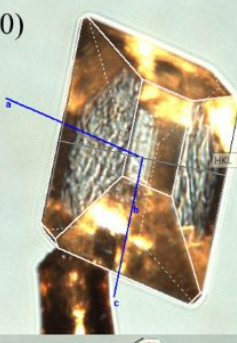

(001)

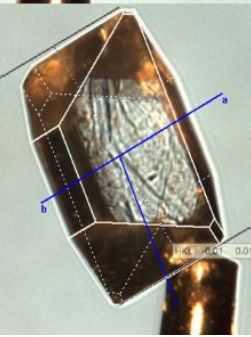

(010)

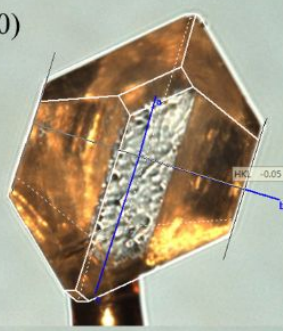

(00-1)

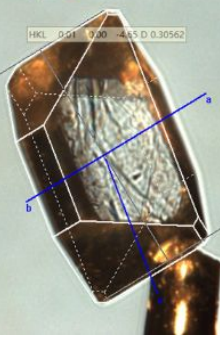

Figure S11. The crystal thickness measurement of Sample 3 along the $a, b$ and $c$-axis.

Table S13. The thickness of sample 3 along the $a, b$ and $c$-axis.

\begin{tabular}{|c|c|c|c|c|c|c|}
\hline Sample & \multicolumn{5}{|c|}{ Distance from the facet to the center of the crystal, D, in the unit of $\mathrm{mm}$} \\
\hline & $(100)$ & $(-100)$ & $(010)$ & $(0-10)$ & $(001)$ & $(00-1)$ \\
\hline $\mathbf{3}$ & 0.215 & 0.210 & 0.279 & 0.284 & 0.314 & 0.306 \\
\hline
\end{tabular}


$(100)$

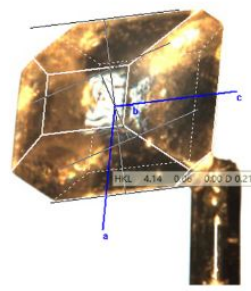

$(0-10)$

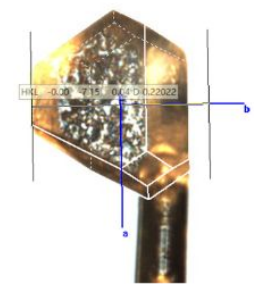

$(-100)$

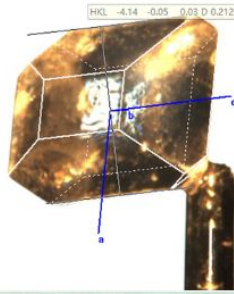

(001)

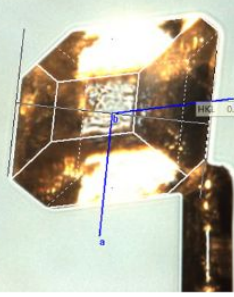

$(010)$

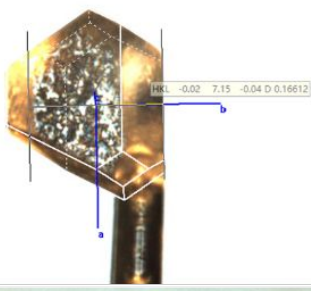

$(00-1)$

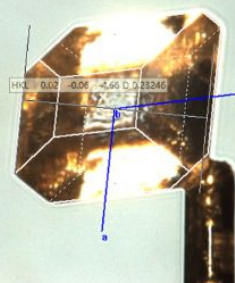

Figure S12. The crystal thickness measurement of Sample 4 along the $a, b$ and $c$-axis.

Table S14. The thickness of sample 4 along the $a, b$ and $c$-axis.

\begin{tabular}{|c|c|c|c|c|c|c|}
\hline Sample & \multicolumn{5}{|c|}{ Distance from the facet to the center of the crystal, D, in the unit of $\mathrm{mm}$} \\
\hline & $(100)$ & $(-100)$ & $(010)$ & $(0-10)$ & $(001)$ & $(00-1)$ \\
\hline $\mathbf{4}$ & 0.216 & 0.212 & 0.166 & 0.220 & 0.242 & 0.232 \\
\hline
\end{tabular}

$(100)$

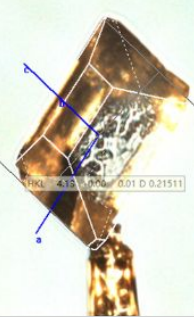

$(0-10)$

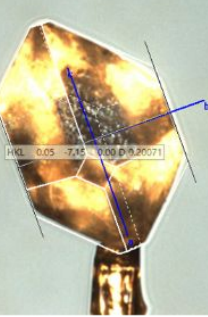

$(-100)$

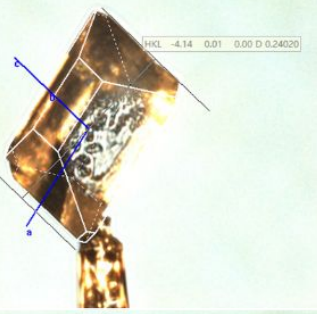

$(001)$

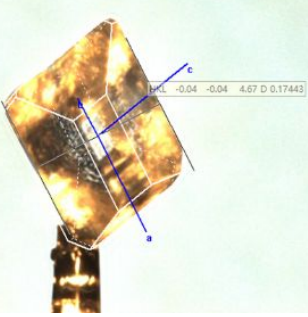

(010)

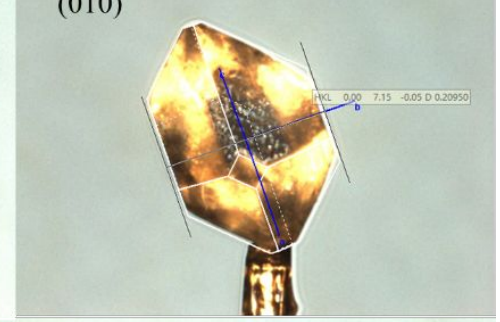

$(00-1)$

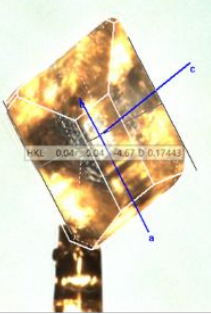

Figure S13. The crystal thickness measurement of Sample 5 along the $a, b$ and $c$-axis.

Table S15. The thickness of sample 5 along the $a, b$ and $c$-axis.

\begin{tabular}{|c|c|c|c|c|c|c|}
\hline Sample & \multicolumn{5}{|c|}{ Distance from the facet to the center of the crystal, D, in the unit of $\mathrm{mm}$} \\
\hline & $(100)$ & $(-100)$ & $(010)$ & $(0-10)$ & $(001)$ & $(00-1)$ \\
\hline $\mathbf{5}$ & 0.215 & 0.240 & 0.210 & 0.201 & 0.174 & 0.174 \\
\hline
\end{tabular}




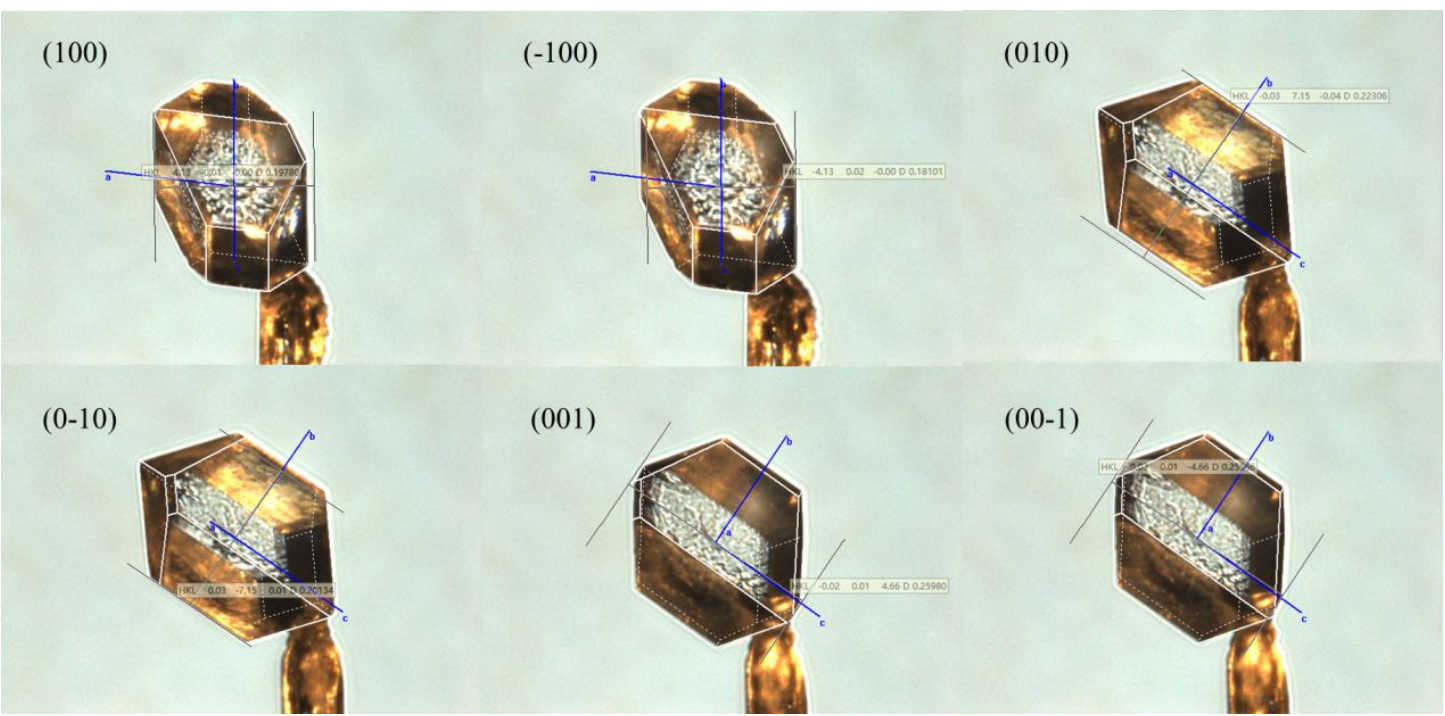

Figure S14. The crystal thickness measurement of Sample 6 along the $a, b$ and $c$-axis.

Table S16. The thickness of sample 6 along the $a, b$ and $c$-axis.

\begin{tabular}{|c|c|c|c|c|c|c|}
\hline Sample & \multicolumn{5}{|c|}{ Distance from the facet to the center of the crystal, D, in the unit of mm } \\
\hline & $(100)$ & $(-100)$ & $(010)$ & $(0-10)$ & $(001)$ & $(00-1)$ \\
\hline $\mathbf{6}$ & 0.198 & 0.181 & 0.223 & 0.201 & 0.260 & 0.256 \\
\hline
\end{tabular}
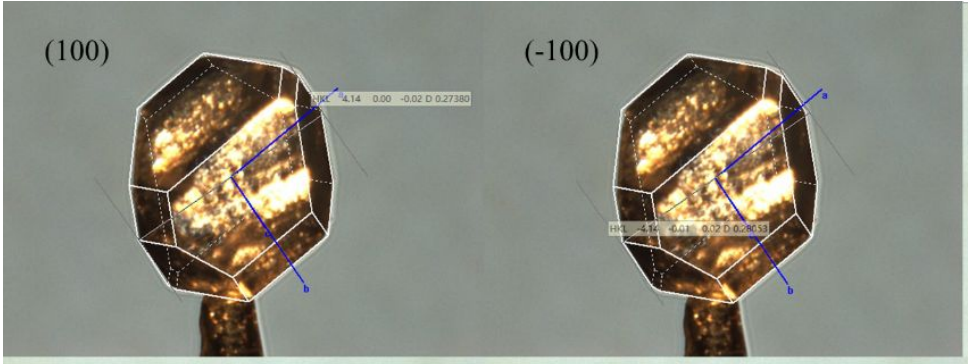

(010)

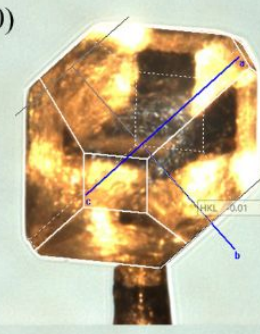

$(0-10)$

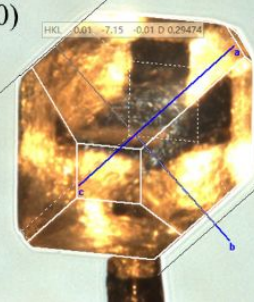

$(001)$

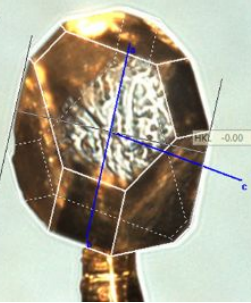

$(00-1)$

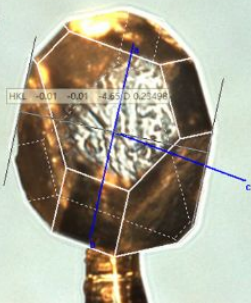

Figure S15. The crystal thickness measurement of Sample 7 along the $a, b$ and $c$-axis.

Table S17. The thickness of sample 7 along the $a, b$ and $c$-axis.

\begin{tabular}{|c|c|c|c|c|c|c|}
\hline sample & \multicolumn{5}{|c|}{ Distance from the facet to the center of the crystal, D, in the unit of $\mathrm{mm}$} \\
\hline & $(100)$ & $(-100)$ & $(010)$ & $(0-10)$ & $(001)$ & $(00-1)$ \\
\hline $\mathbf{7}$ & 0.274 & 0.281 & 0.258 & 0.295 & 0.239 & 0.235 \\
\hline
\end{tabular}


(100)

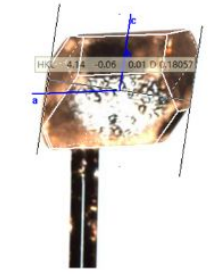

(0-10)

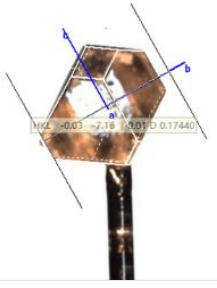

$(-100)$

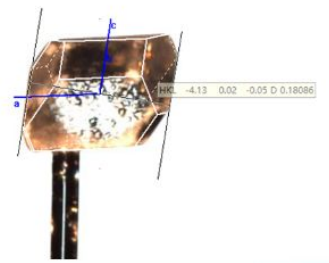

$(001)$

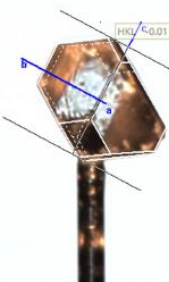

$(010)$

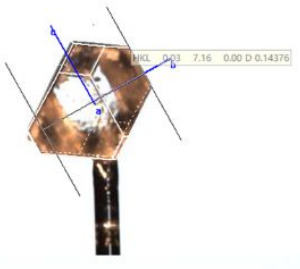

$(00-1)$

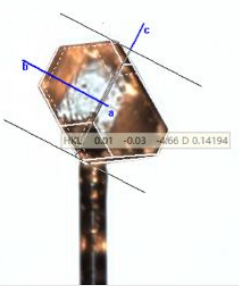

Figure S16. The crystal thickness measurement of Sample 8 along the $a, b$ and $c$-axis.

Table S18. The thickness of sample 8 along the $a, b$ and $c$-axis.

\begin{tabular}{|c|c|c|c|c|c|c|}
\hline Sample & \multicolumn{5}{|c|}{ Distance from the facet to the center of the crystal, D, in the unit of $\mathrm{mm}$} \\
\hline & $(100)$ & $(-100)$ & $(010)$ & $(0-10)$ & $(001)$ & $(00-1)$ \\
\hline $\mathbf{8}$ & 0.181 & 0.181 & 0.144 & 0.174 & 0.146 & 0.142 \\
\hline
\end{tabular}

Table S19. The summary of the thickness of the HMX crystals grown in DMSO along the $a$ (D1), $b$ (D2) and $c$-axis (D3) and their ratios.

\begin{tabular}{|c|c|c|c|c|c|}
\hline Sample & \multicolumn{3}{|c|}{ Thickness (mm)* } & \multicolumn{2}{c|}{ Ratio } \\
\hline & $\mathbf{D 1}$ & $\mathbf{D 2}$ & $\mathbf{D 3}$ & D1 : D3 & D2 : D3 \\
\hline $\mathbf{1}$ & 0.813 & 0.752 & 0.688 & 1.18 & 1.09 \\
\hline $\mathbf{2}$ & 0.653 & 0.781 & 0.821 & 0.79 & 0.95 \\
\hline $\mathbf{3}$ & 0.425 & 0.563 & 0.620 & 0.69 & 0.91 \\
\hline $\mathbf{4}$ & 0.428 & 0.386 & 0.475 & 0.90 & 0.81 \\
\hline $\mathbf{5}$ & 0.455 & 0.410 & 0.349 & 1.31 & 1.18 \\
\hline $\mathbf{6}$ & 0.379 & 0.424 & 0.516 & 0.73 & 0.82 \\
\hline $\mathbf{7}$ & 0.554 & 0.552 & 0.474 & 1.17 & 1.17 \\
\hline $\mathbf{8}$ & 0.361 & 0.318 & 0.288 & 1.25 & 1.10 \\
\hline \multicolumn{3}{|c|}{ Average } & 1.00 & 1.00 \\
\hline
\end{tabular}

* D1 $=\mathbf{D}(100)+\mathbf{D}(-100), \mathbf{D} 2=\mathbf{D}(010)+\mathbf{D}(0-10)$ and $\mathbf{D 3}=\mathbf{D}(001)+\mathbf{D}(00-1)$

Table S20. The thickness of the HMX crystals grown in DMSO along the facet directions, in the unit of $\mathrm{mm}$.

\begin{tabular}{|l|c|c|c|c|c|c|c|}
\hline & $(\mathbf{0 1 1})$ & $\mathbf{( 0 1 - 1 )}$ & $\mathbf{( 1 1 0 )}$ & $\mathbf{( 1 - 1 0 )}$ & $\mathbf{( 0 2 0})$ & $\mathbf{( 1 0 - 1 )}$ & $\mathbf{( 1 0 1 )}$ \\
\hline $\mathbf{1}$ & 0.493 & 0.672 & 0.751 & 0.662 & 0.747 & - & 0.795 \\
\hline $\mathbf{2}$ & 0.743 & 0.648 & 0.499 & 0.655 & 0.786 & 1.119 & 0.798 \\
\hline $\mathbf{3}$ & 0.480 & 0.556 & 0.420 & 0.344 & 0.565 & 0.785 & 0.565 \\
\hline $\mathbf{4}$ & 0.479 & 0.324 & 0.413 & 0.335 & 0.392 & - & 0.464 \\
\hline $\mathbf{5}$ & 0.275 & 0.328 & 0.413 & 0.396 & 0.401 & 0.601 & 0.462 \\
\hline $\mathbf{6}$ & 0.413 & 0.459 & 0.355 & 0.308 & 0.427 & - & 0.439 \\
\hline $\mathbf{7}$ & 0.381 & 0.430 & 0.458 & 0.497 & 0.553 & 0.736 & 0.479 \\
\hline
\end{tabular}


Supplementary information

8

0.278

0.224

0.342

0.303

0.327

0.471

0.324 
Part 2. Area measurement of the facets of the HMX crystals grown in acetone and DMSO.

Table S21. The area of the exposed facets for HMX grown in acetone in the unit of $\mathrm{mm}^{2}$.

\begin{tabular}{|c|c|c|c|c|c|c|c|c|}
\hline Facet & Sample 1 & Sample 2 & Sample 3 & Sample 4 & Sample 5 & Sample 6 & Sample 7 & Sample 8 \\
\hline$(011)$ & 0.057818 & 0.046070 & 0.098819 & 0.184638 & 0.235190 & 0.023694 & 0.019009 & 0.009574 \\
\hline$(0-1-1)$ & 0.200672 & 0.059166 & 0.059285 & 0.211313 & 0.139769 & 0.023883 & 0.020850 & 0.010623 \\
\hline$(01-1)$ & 0.181184 & 0.129760 & 0.161156 & 0.109879 & 0.149764 & 0.022155 & 0.022303 & 0.007269 \\
\hline$(0-11)$ & 0.323981 & 0.142861 & 0.121627 & 0.136553 & 0.055472 & 0.022963 & 0.024312 & 0.006440 \\
\hline$(110)$ & 0.082749 & 0.024939 & 0.016255 & 0.081382 & 0.058069 & 0.020011 & 0.007148 & 0.003839 \\
\hline $\mathbf{( - 1 - 1 0 )}$ & 0.057220 & 0.047809 & 0.064990 & 0.071085 & 0.018801 & 0.025424 & 0.006232 & 0.003842 \\
\hline$(1-10)$ & 0.101472 & 0.070164 & 0.087417 & 0.052521 & 0.025267 & 0.025520 & 0.006480 & 0.002493 \\
\hline$(-110)$ & 0.127010 & 0.047299 & 0.038683 & 0.062815 & 0.065759 & 0.020782 & 0.007579 & 0.000443 \\
\hline$(020)$ & 0.151451 & 0.054379 & 0.035319 & 0.036514 & 0.000266 & 0.016719 & 0.004057 & 0.005398 \\
\hline$(0-20)$ & 0.021175 & 0.017180 & 0.029669 & 0.017587 & 0.143677 & 0.011093 & 0.002963 & 0.006544 \\
\hline$(10-1)$ & - & - & - & - & 0.001587 & 0.001349 & 0.002220 & 0.003521 \\
\hline$(-101)$ & - & - & - & - & 0.000319 & 0.000659 & 0.002033 & 0.005617 \\
\hline
\end{tabular}

Table S22. The area of the exposed facets for HMX grown in DMSO in the unit of $\mathrm{mm}^{2}$.

\begin{tabular}{|c|c|c|c|c|c|c|c|c|}
\hline Facet & Sample 1 & Sample 2 & Sample 3 & Sample 4 & Sample 5 & Sample 6 & Sample 7 & Sample 8 \\
\hline$(011)$ & 0.293671 & 0.174590 & 0.105141 & 0.039363 & 0.091939 & 0.061215 & 0.120975 & 0.037905 \\
\hline$(0-1-1)$ & 0.381561 & 0.171590 & 0.109314 & 0.038333 & 0.100225 & 0.076487 & 0.146559 & 0.044141 \\
\hline$(01-1)$ & 0.156074 & 0.228061 & 0.072024 & 0.100136 & 0.068056 & 0.055488 & 0.096123 & 0.057683 \\
\hline$(0-11)$ & 0.235681 & 0.230876 & 0.081032 & 0.101674 & 0.074815 & 0.052075 & 0.118039 & 0.064464 \\
\hline$(110)$ & 0.174864 & 0.345975 & 0.127282 & 0.036835 & 0.056580 & 0.081569 & 0.093698 & 0.024354 \\
\hline$(-1-10)$ & 0.143948 & 0.307275 & 0.107588 & 0.071742 & 0.041140 & 0.073966 & 0.097426 & 0.028510 \\
\hline$(1-10)$ & 0.193406 & 0.176654 & 0.158673 & 0.111406 & 0.045760 & 0.087561 & 0.072397 & 0.041399 \\
\hline$(-110)$ & 0.233367 & 0.209922 & 0.170756 & 0.073696 & 0.064415 & 0.115535 & 0.080088 & 0.035012 \\
\hline$(020)$ & 0.110757 & 0.088366 & 0.025068 & 0.066945 & 0.019535 & 0.028215 & 0.042105 & 0.023039 \\
\hline$(0-20)$ & 0.054825 & 0.052284 & 0.033787 & 0.030297 & 0.028395 & 0.039552 & 0.018172 & 0.010661 \\
\hline$(10-1)$ & - & 0.001594 & 0.000560 & - & 0.001863 & - & 0.005098 & 0.000791 \\
\hline$(-101)$ & - & 0.001119 & 0.001758 & - & 0.001069 & - & 0.001298 & 0.001628 \\
\hline$(101)$ & 0.021998 & 0.007632 & 0.002969 & 0.008466 & 0.004929 & 0.028911 & 0.032679 & 0.008581 \\
\hline$(-10-1)$ & 0.010428 & 0.015143 & 0.011196 & 0.012041 & 0.001812 & 0.002888 & 0.022829 & 0.010389 \\
\hline
\end{tabular}

